\title{
Increased syndecan-1 in serum is related to early nephropathy in type 1 diabetes mellitus patients
}

\author{
K. Svennevig • S. O. Kolset • H.-J. Bangstad
}

Received: 17 February 2006 / Accepted: 24 April 2006 / Published online: 11 July 2006

(C) Springer-Verlag 2006

To the Editor: Hyperglycaemia is associated with increased inflammatory activity, endothelial dysfunction and microvascular complications in patients with type 1 diabetes mellitus. Low-grade inflammation and changes in functions of different constituents of the capillary wall may contribute to the development of complications.

The endothelium is covered by a protective glycocalyx consisting of proteoglycans, hyaluronan, glycoproteins and plasma proteins. The transmembrane proteoglycan syndecan-1 is a component of this cell coat. Through its glycosaminoglycan chains, syndecan-1 has the ability to bind numerous ligands and by such interactions to modulate important cellular functions like receptor binding and regulation of inflammation [1].

The syndecan-1 ectodomain can be released from the endothelial cell surface by a highly regulated proteolytic cleavage at a juxtamembrane site of the protein core. Syndecan-1 concentrations found in sera from healthy individuals are low. Shed syndecan-1 levels are often increased in tissue injury and certain cancers.

In this study we tested for associations between early kidney damage, as evidenced by persistent microalbuminuria, and shedding of syndecan-1, measured by determining serum concentrations of soluble syndecan-1 in patients with type 1 diabetes mellitus.

\footnotetext{
K. Svennevig $\cdot$ H.-J. Bangstad

Department of Pediatrics, Woman and Child Division,

Ullevål University Hospital,

Oslo, Norway

K. Svennevig $(\bowtie) \cdot$ S. O. Kolset

Department of Nutrition, Institute of Basic Medical Sciences, University of Oslo, P.O. Box 1046, Blindern, 0316 Oslo, Norway

email: katja.fjeldstad@biokjemi.uio.no
}

In an initial study, patients with type 1 diabetes mellitus were recruited from Aker University Hospital and nearby paediatric Departments [2]. The inclusion criteria in this study were persistent microalbuminuria, defined as an AER between 15 and $200 \mu \mathrm{g} / \mathrm{min}$ in at least two out of three overnight urine samples taken during 1 year, and diabetes duration of $>5$ years. A total of 18 patients took part in this randomised prospective study investigating the importance of improved blood glucose control and intensive insulin treatment on ultrastructural glomerular changes. The parameters registered for all patients were age, sex, diabetes duration, GFR, AER, $\mathrm{HbA}_{1 \mathrm{c}}$, and blood pressure. The patients had neither clinical nephropathy nor proliferative retinopathy, and all except one patient had blood pressure $<140 / 90 \mathrm{mmHg}$ at the start of the study.

In the investigation reported here, the syndecan-1 concentration in 17 of the 18 patients from whom serum samples at the start of the study were available were compared with samples from 12 matched normoalbuminuric type 1 diabetes mellitus patients taken in the same period. Age, duration of diabetes, sex ratio, and $\mathrm{HbA}_{1 \mathrm{c}}(p=0.07)$, were similar in the two groups. We recruited 24 healthy control subjects without diabetes from healthy students and staff in the Department of Nutrition. All subjects in this study gave informed consent and the study was approved by the Regional Ethics Committee.

For the quantitative determination of soluble syndecan-1 in serum samples a commercially available ELISA kit was used (Diaclone Research, Besançon, France).

$\mathrm{HbA}_{1 \mathrm{c}}$ was analysed by an HPLC method (Biorad, Richmond, CA, USA). The normal range is $4.3-6.1 \%$, inter-assay CV 3\%. The mean of three to four measurements per patients during 1 year is given.

The Wilcoxon rank-sum test for difference in medians was applied for comparison between groups. Two-tailed 
tests were used. Correlations were tested with Spearman's rank correlation coefficient. Results are presented as median and $95 \%$ CI. The level of statistical significance was set at 5\% (NCSS, Kaysville, UT, USA).

In microalbuminuric type 1 diabetes mellitus patients the median syndecan- 1 serum concentration was $113 \mu \mathrm{g} / \mathrm{ml}$ (CI 89-143), which was significantly increased $(p<0.0001)$ compared with that in patients with normoalbuminuria $(39 \mu \mathrm{g} / \mathrm{ml}, \mathrm{CI} 25-54)$ and healthy individuals $(34 \mu \mathrm{g} / \mathrm{ml}, \mathrm{CI}$ $10-40, p<0.00001$ ) (Fig. 1). The soluble syndecan-1 concentrations did not show a statistically significant correlation with age, diabetes duration, sex, blood pressure, basement membrane thickness, GFR or $\mathrm{HbA}_{1 \mathrm{c}}$.

This study demonstrates that serum syndecan- 1 concentrations are significantly increased in microalbuminuric compared with normoalbuminuric type 1 diabetes mellitus patients and healthy controls. To our knowledge this is the first study to measure the serum level of soluble syndecan-1 in type 1 diabetes mellitus patients. The patients with microalbuminuria are hyperfiltrating, thus reduced GFR does not explain the elevated syndecan-1 level in our series.

Our result can be explained by previous studies on the connection between hyperglycaemia, diabetes mellitus, endothelial dysfunction and microalbuminuria. Hyperglycaemia has been identified as the primary factor in the development of diabetic complications. Toxic effects of oxidants, hyperosmolarity, or glycation products stimulate expression of several cytokines and alterations in cell signalling pathways [3]. The endothelial cells are the first cells to be affected by hyperglycaemia. Endothelial dysfunction has been documented in type 1 diabetes mellitus patients in whom clinically manifest microalbuminuria is present, and it represents a link to the progression of nephropathy [4].

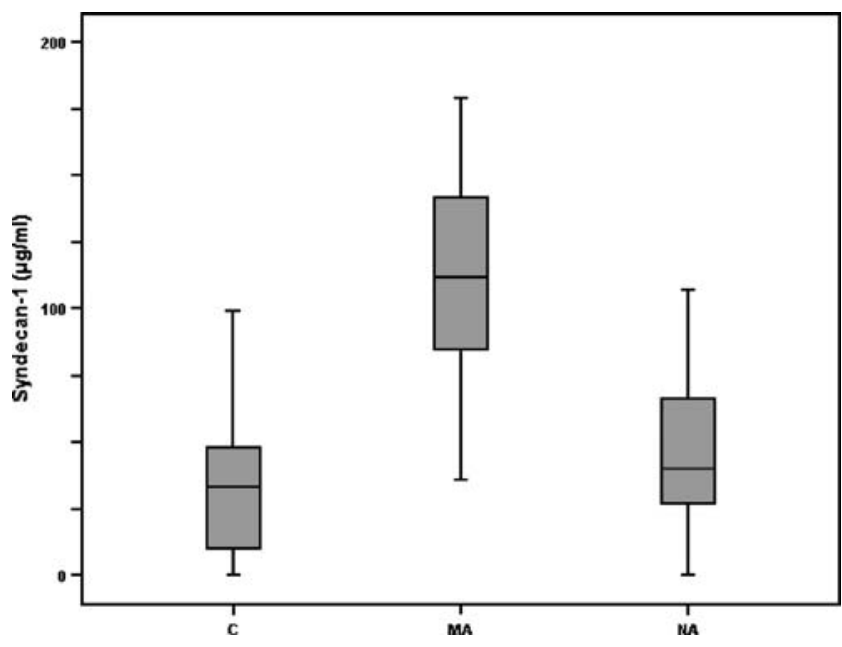

Fig. 1 Syndecan-1 concentrations in serum from subjects with type 1 diabetes mellitus and microalbuminuria $(M A, n=17)$ or normoalbuminuria $(N A, n=12)$ and in serum from control subjects $(C, n=24)$
Type 1 diabetes mellitus is associated with increased cytokine-mediated inflammation [5]. In agreement with our results it has been shown that components of the endothelial glycocalyx are shed in response to inflammation [6].

The role of soluble syndecan- 1 in relation to diabetes complications has not been determined. The syndecan-1 ectodomains can act as soluble effector molecules [1]. Experiments in animal models suggest that absence of the glycocalyx on the endothelial cell surface leads to increased capillary permeability, enhanced interaction between leucocytes and endothelial cells, increased angiogenesis and inflammatory responses [7]. In addition glycosaminoglycan chains attach extracellular superoxide dismutase to the vascular endothelial cell surface. Loss of proteoglycan on the cell surface can probably decrease local antioxidant activity, increasing the susceptibility of tissues to oxidant-mediated tissue damage [8]. High glucose concentrations are known to reduce proteoglycan production in glomerular endothelial cells, indicating that the shed syndecan-1 is not replaced on the cell surface by increased synthesis [9].

In the present study, the finding that increased serum levels of syndecan-1 are related to microalbuminuria in patients with type 1 diabetes mellitus further supports the notion that syndecan-1 plays a role in the pathogenesis of kidney complications in diabetes. Removing syndecan-1 from the cell coat may affect permeability of the endothelium, leading to leakage of proteins and microalbuminuria.

On the basis of the data presented, modulation of syndecan-1 expression and the sheddases involved could be one possible way to influence glomerular damage in type 1 diabetes mellitus. To replicate and confirm our finding, syndecan-1 should be measured in serum from a greater number of type 1 diabetes mellitus patients. In addition further experiments are needed to identify the proteases involved in syndecan-1 shedding.

Acknowledgements This study was supported by grants from Helse-Øst and the Throne Holst foundation.

\section{References}

1. Gotte M, Echtermeyer F (2003) Syndecan-1 as a regulator of chemokine function. Scientific World Journal 3:1327-1331

2. Bangstad HJ, Osterby R, Dahl-Jorgensen K, Berg KJ, Hartmann A, Hanssen KF (1994) Improvement of blood glucose control in IDDM patients retards the progression of morphological changes in early diabetic nephropathy. Diabetologia 37:483-490

3. Sheetz MJ, King GL (2002) Molecular understanding of hyperglycemia's adverse effects for diabetic complications. JAMA 288:2579-2588

4. Creager MA, Luscher TF, Cosentino F, Beckman JA (2003) Diabetes and vascular disease: pathophysiology, clinical consequences, and medical therapy. Part I. Circulation 108:1527-1532

5. Basu S, Larsson A, Vessby J, Vessby B, Berne C (2005) Type 1 diabetes is associated with increased cyclooxygenase- and cytokine-mediated inflammation. Diabetes Care 28:1371-1375 
6. Mulivor AW, Lipowsky HH (2004) Inflammation- and ischemiainduced shedding of venular glycocalyx. Am J Physiol Heart Circ Physiol 286:H1672-H1680

7. Mulivor AW, Lipowsky HH (2002) Role of glycocalyx in leukocyte-endothelial cell adhesion. Am J Physiol Heart Circ Physiol 283:H1282-H1291
8. Yamamoto M, Hara H, Adachi T (2001) Nitric oxide and its decomposed derivatives decrease the binding of extracellular-superoxide dismutase to the endothelial cell surface. FEBS Lett 505:296-300

9. Ha TS, Duraisamy S, Faulkner JL, Kasinath BS (2004) Regulation of glomerular endothelial cell proteoglycans by glucose. J Korean Med Sci 19:245-252 